\title{
Review Article \\ Effectiveness of Acupuncture for Early Recovery of Bowel Function in Cancer: A Systematic Review and Meta-Analysis
}

\author{
Yi-Hua Liu, ${ }^{1,2}$ Guang-Tong Dong, ${ }^{1}$ Yang Ye, ${ }^{2}$ Jia-Bin Zheng, ${ }^{1}$ Ying Zhang, \\ Hong-Sheng Lin, ${ }^{1}$ and Xue-Qian Wang ${ }^{1}$ \\ ${ }^{1}$ Department of Oncology, Guang'anmen Hospital, China Academy of Chinese Medical Sciences, No. 5 Beixiange Street, \\ Xicheng District, Beijing 100053, China \\ ${ }^{2}$ Beijing University of Chinese Medicine, No. 11 North Third Ring Road, Chaoyang District, Beijing 100029, China
}

Correspondence should be addressed to Hong-Sheng Lin; drlinhongsheng@126.com

Received 8 March 2017; Revised 10 July 2017; Accepted 14 September 2017; Published 20 December 2017

Academic Editor: Kieran Cooley

Copyright (C) 2017 Yi-Hua Liu et al. This is an open access article distributed under the Creative Commons Attribution License, which permits unrestricted use, distribution, and reproduction in any medium, provided the original work is properly cited.

\begin{abstract}
Objectives. The aim of this study was to evaluate the effects of acupuncture therapy to reduce the duration of postoperative ileus (POI) and to enhance bowel function in cancer patients. Methods. A systematic search of electronic databases for studies published from inception until January 2017 was carried out from six databases. Randomized controlled trials (RCTs) involving the use of acupuncture and acupressure for POI and bowel function in cancer patients were identified. Outcomes were extracted from each study and pooled to determine the risk ratio and standardized mean difference. Results. 10 RCTs involving 776 cancer patients were included. Compared with control groups (no acupuncture, sham acupuncture, and other active therapies), acupuncture was associated with shorter time to first flatus and time to first defecation. A subgroup analysis revealed that manual acupuncture was more effective on the time to first flatus and the time to first defecation; electroacupuncture was better in reducing the length of hospital stay. Compared with control groups (sham or no acupressure), acupressure was associated with shorter time to first flatus. However, GRADE approach indicated a low quality of evidence. Conclusions. Acupuncture and acupressure showed large effect size with significantly poor or inferior quality of included trials for enhancing bowel function in cancer patients after surgery. Further well-powered evidence is needed.
\end{abstract}

\section{Introduction}

Bowel dysfunction has been found to be closely related to worse postoperative quality of life, which is regarded as a major outcome measure in surgical oncology $[1,2]$. A temporary impairment of bowel motility lasting within 3-5 days, known as postoperative ileus (POI), is expected after any major surgical procedure, including cancer surgery [3-5]. The clinical manifestations of POI include abdominal pain, nausea, vomiting, and delay in the passage of flatus and stool [6]. A commonly used clinical end point of POI was the time to recovery of bowel function; the primary therapeutic goal in the treatment of POI is to decrease the time to first flatus and defecation [7]. POI is associated with discomfort experienced by patients, delayed patient recovery, prolonged length of hospital stay, and increased healthcare costs [8]. Epidemiological evidence supports the fact that POI is the second most common reason for hospital readmission following surgery; the incidence of POI is 10 to $30 \%[9,10]$. The annual economic impact of POI management has been estimated to be over 1.5 billion dollars [11].

Since POI is one of the major causes for the delayed recovery of bowel function following cancer resection [12], pharmacological and nonpharmacological therapies have been directed toward alleviating POI [13]. Pharmacological agents such as cyclooxygenase 2 (COX-2) inhibitors, ghrelin agonists, and opioid agonists always bring side effects, such as cardiovascular adverse events and immunosuppressive effects [14]. The laparoscopic technique has been proven to reduce the incidence of POI, while the costs restrict its application. Indeed, no drugs or interventions to prevent POI have been approved by the US Food and Drug Administration [15]. Recently, the Enhanced Recovery after Surgery (ERAS) programs have proposed that measures should aim more at 
the prevention of POI than its treatment $[16,17]$, of which acupuncture has become a promising option.

As a nonpharmacological intervention, acupuncture is commonly used for various functional gastrointestinal disorders. A preclinical animal study demonstrated that acupuncture could promote the recovery time of POI by activating the vagus nerve to improve the gastrointestinal tract transit function [18]. Moreover, acupuncture has been widely practiced as an appropriate adjunctive treatment for cancer symptoms $[19,20]$. Many researchers have investigated the efficacy of acupuncture therapy for postoperative recovery in cancer patients with POI. However, whether acupuncture has a definite therapeutic effect on POI in cancer patients remains controversial. Two previous systematic reviews broadly for cancer care have examined this issue but did not use metaanalysis $[19,21]$. To the best of our knowledge, a meta-analysis has never been performed to address this issue. Hence, we conducted this systematic review and meta-analysis to comprehensively assess the effectiveness of acupuncture and acupressure in preventing POI and enhancing bowel function in cancer patients.

\section{Materials and Methods}

2.1. Protocol and Registration. This meta-analysis follows Preferred Reporting Items for Systematic Reviews and MetaAnalyses Statement (PRISMA) guidelines [22]. The registered study protocol is available on PROSPERO database (identification number: CRD 42016049633).

2.2. Data Sources and Search Strategy. The following databases were searched from inception until January 2017: PubMed, EMBASE, Cochrane Central, VIP database for Chinese Technical Periodicals, China National Knowledge Infrastructure database, and Wanfang database. The complete manuscripts of all relevant studies published in English and Chinese were retrieved. The key search terms included [cancer OR tumor OR neoplasm OR oncology] AND [acupuncture OR electroacupuncture OR acupressure] AND [ileus OR intestinal obstruction OR gastrointestinal dysfunction]. The search strategy was modified to suit each database. Additionally, we searched the following databases of ongoing trials: the WHO International Clinical Trials Registry Platform and clinicaltrials.gov. The reference lists of relevant reviews were cross-examined to avoid the risk of missing eligible RCTs.

2.3. Inclusion Criteria. The inclusion criteria were as follows: (1) participants: participants underwent surgery were adults aged 18 years or older who were formally diagnosed with cancer. There were no limitations on race, gender, or tumor type. (2) Interventions: manual acupuncture (MA), electroacupuncture (EA), and acupressure were defined as the only forms. Studies that evaluated auricular acupuncture, moxibustion, microacupuncture, and acupuncture point injection were excluded (the methodology and principles in mechanism differ from acupuncture therapy). (3) Controls: no acupuncture, sham acupuncture, and other active control therapies were considered, as no precise definition of placebo acupuncture exists. RCTs comparing acupuncture directly with different types of herbal medications were excluded from this study. (4) Outcomes: the primary outcomes used in this systematic review were time to first flatus and time to defecation, which have been shown to be clinical hallmarks of POI [7]. Secondary outcomes included time to first bowel sounds, opioids consumption, visual analog scale (VAS) pain score, the risk of POI, and length of hospital stay [7, 33]. Studies that did not report at least two aggregate outcomes were excluded. Any adverse events (AE) were measured (if available). (5) Studies: eligible studies had to meet the criteria as follows: only prospective RCTs that evaluated acupuncture to treat POI or bowel dysfunction in cancer patients following surgery were considered; published in a peer-reviewed journal; had original data being independent of other studies. Any conflicts on the eligibility of studies were resolved by consulting a senior researcher (HSL).

2.4. Data Extraction. Two reviewers (Yi-Hua Liu and Yang Ye) screened all the records independently. Excluded studies and exclusive reasons were listed. Relevant articles were sorted and cross-examined. The relevant information was extracted using a standardized data extraction form which included first author and publication year, country, study design, baseline characteristics of patients, tumor type, experimental and control type, treatment course, and outcome assessment. The details of intervention and control group were extracted according to the Standards for Reporting Interventions in Controlled Trials of Acupuncture (STRICTA) checklist items. A checklist included theory of acupuncture, needle depth, needle location, name and number of acupuncture points selected, "Deqi" sensation, and duration of treatment sessions [34]. Any discrepancies were discussed and resolved by agreement or by consulting a senior researcher (HSL).

2.5. Quality Assessment. The methodological quality was assessed according to the Cochrane Handbook version 5.1.0 $[34,35]$. The risk of bias quality was assessed based on seven items: random sequence generation, allocation concealment, blinding of participants and personnel, blinding of outcome assessment, incomplete outcome data, selective reporting, and other bias. After assessing all the domains, the risk of bias was categorized as three levels: low risk, unclear risk, and high risk. Moreover, the Grading of Recommendations Assessment, Development and Evaluation (GRADE) approach was used to facilitate the overall quality of each outcome [36]. The evidence of GRADE was generated using GRADEpro version 3.6 software. Any discrepancies were discussed and resolved by agreement or by consulting a senior researcher (HSL).

2.6. Statistical Analysis. Effect sizes were presented as risk ratio (RR) and standardized mean difference (SMD). Dichotomous data were presented as risk ratio (RR) with $95 \%$ confidence interval (CI); the standardized mean difference (SMD) with 95\% CI was used for effect estimates because outcome measurements are on the various scales. The clinical significance for the SMD was rated $(<0.40,0.40 \sim 0.70,>0.70$ was, respectively, regarded as low, moderate, and large) 


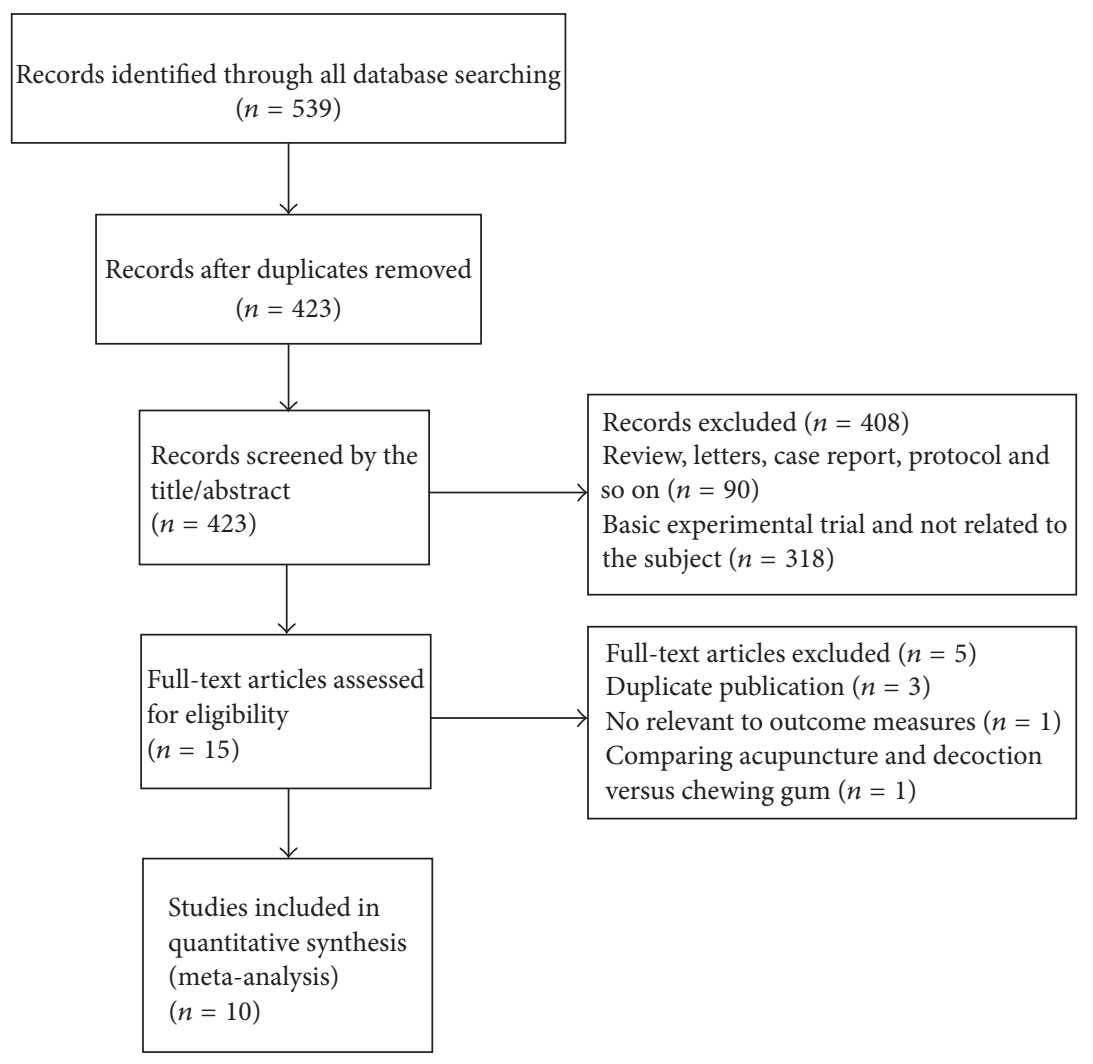

FIGURE 1: Flow diagram of studies included in the review.

according to Cohen's interpretation of effect size. Cochrane $Q$ test and $I^{2}$ (25\%, 50\%, and 75\% were, respectively, regarded as low, moderate, and high heterogeneity) were used to assess the heterogeneity; $I^{2}<50 \%$ indicates acceptable heterogeneity [37]. The random effect models were used to give an estimate of the effect because heterogeneity between multistudies and wider intervals has to be considered. Subgroup analysis was designed to investigate the effect of different acupuncture approaches including MA and EA. Sensitivity analysis was carried out for primary outcomes by removing one study a time to investigate the robustness of the review findings. Funnel plots were generated to examine the publication bias and assessed by using Begg's and Egger's tests (if at least 10 trials were available for a meta-analysis) [38]. Cumulative metaanalyses were conducted to analyze how the pooled effect has changed over time. Data analysis was applied using RevMan 5.3 (Cochrane Collaboration) and Stata 12.0 (StataCorp). All $P$ values were two sided.

\section{Results}

3.1. Search Results. A total of 539 articles were identified in the initial research. Among them, 116 articles were excluded because they were duplicate trials. After screening based on titles and abstracts, 408 were excluded because they were nonclinical trials, case reports, reviews, protocols, or not related to the subject. Of the remaining 15 articles, by reading the full text, 10 articles met our inclusion criteria [23-32].
Flow diagram of the screening process is summarized in Figure 1.

3.2. Characteristics of Included Studies. The basic characteristics of the included trials are listed in Table 1. All of the trials were RCTs published from 2008 to 2015. One trial was conducted in the United State [23] and nine conducted in China [24-32].

3.2.1. Participants. A total of 776 cancer patients with sample sizes ranging from 39 to 165 were included in 10 RCTs. Among them, 358 patients received acupuncture treatments, and 418 patients were treated with control. The median age of patients ranged from 53.1 to 68.5. Patients with structural abnormalities were excluded in each trial. Underlying malignancies included gastric cancer [24, 32], colorectal cancer [25-31], and miscellaneous cancer [23].

3.2.2. Interventions. MA was used in five trials $[23,24,27,29$, $31]$, EA was used in three trials $[25,28,30]$, and acupressure was used in two trials $[26,32]$. All trials mentioned the starting time of the intervention. The most commonly used acupuncture points were ST-36 (ten trials) and SP-6 (five trials). Total treatment sessions ranged from 3 days to 10 days, and intervention dose ranged from $3 \mathrm{~min}$ to $45 \mathrm{~min}$.

3.2.3. Outcome Measures. Most trials adopted the time to first flatus and time to first defecation as the primary 


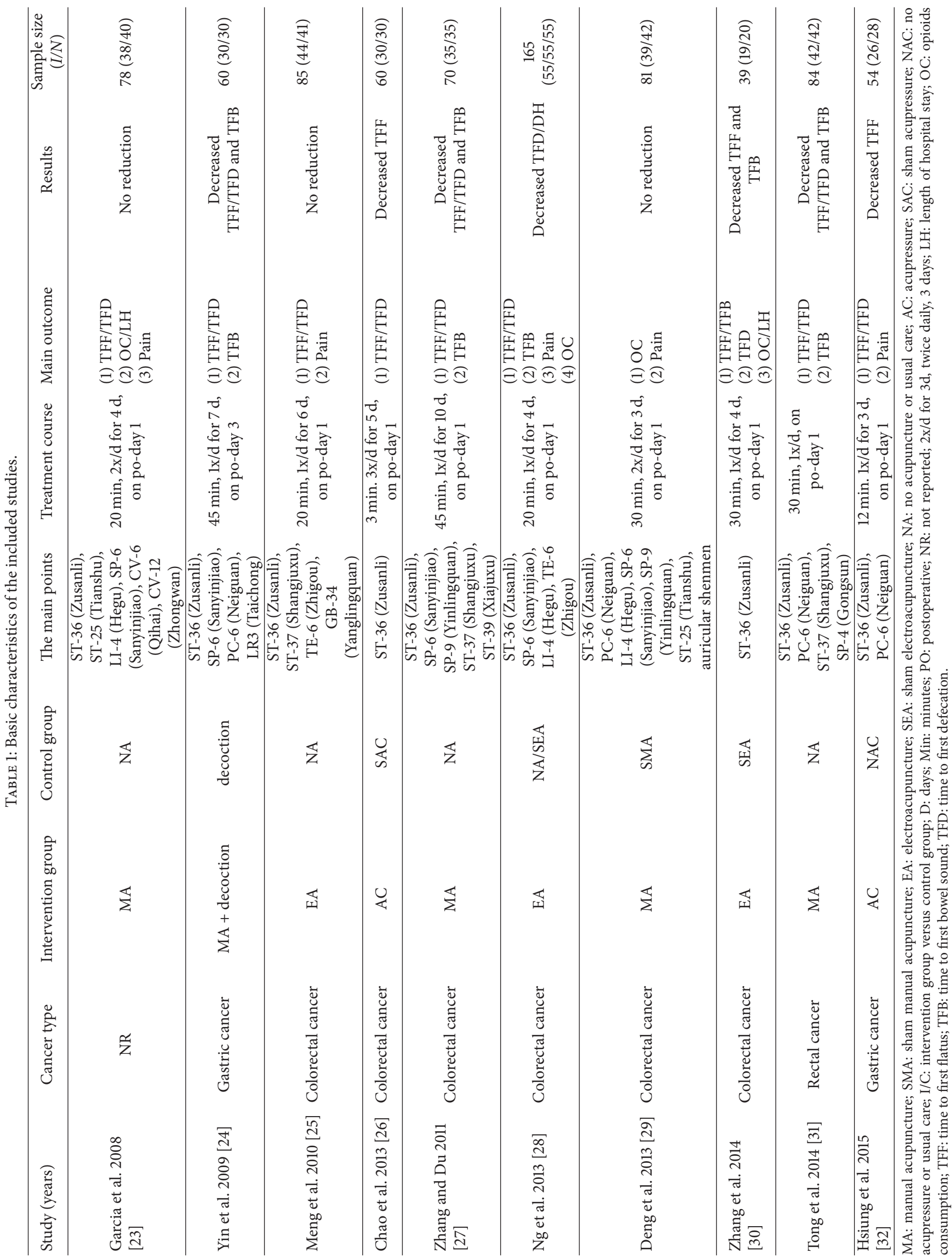


outcome (9/10). For the secondary outcomes, four trials evaluated VAS pain score $[23,25,28,29]$ and opioids consumption [23, 28-30]. Three trials evaluated the risk of POI $[23,25,28]$, the length of hospital stay $[23,28,30]$, and time to first bowel sound [27, 30, 31]. Seven trials concluded the efficacy of acupuncture and acupressure on the improvement of primary outcome [24, 26-28, 30-32], while three trials suggested no effects $[23,25,29]$. For studies with two control groups, we divided the shared intervention evenly between groups as described in the Cochrane Handbook [37]. The details of acupuncture therapy assessed by STRICTA were presented in Supplementary File 3.

3.3. Risk of Bias and Level of Evidence. Although all ten trials reported that they utilized randomized control procedures, only seven trials described the generation of random sequences $[25,26,28-32]$ while five trials described the method of allocation concealment $[25,28-30,32]$. In the trials comparing acupuncture with sham or no acupuncture that did not insert needles into skin, patients blinding was not possible and likely to be a risk in most RCTs. In most of the studies, the use of intention-to-treat analysis was unclear. One trial [25] reported a number of dropouts (10/85); due to the dropouts evenly distributed across intervention and control group, the rate below $15 \%$ would be unlikely to affect the estimates. The total quality of the data was low according to Cochrane risk of bias tools. A summary of risks of bias is presented in Figure 2. The GRADE approach was used to quantify the effects and level of evidence. According to the GRADE assessment, the quality of evidence for most outcomes was from very low to low. The imprecision domain of outcomes was downgraded due to methodological limitations and small sample sizes. The summary of evidence generated using GRADEPRO is presented in Table 2.

3.4. Meta-Analysis Results. We combined the data using a random effect model in the following comparison: (1) acupuncture (MA and EA) versus control; (2) acupressure versus control.

\subsubsection{Effects of Acupuncture Treatment}

Time to First Flatus. Seven trials contributed to the combined calculation of this outcome [23-25, 27, 28, 30, 31]. Pooled results indicated that acupuncture treatment was associated with a significant benefit in time to first flatus ( $S M D=-0.82$, 95\% CI: -1.47 to $-0.17, P=0.01 ; I^{2}=92 \%$ ) (Figure 3 ). A subgroup analysis was conducted to explore whether heterogeneity could be partially explained by different types of acupuncture treatment. Our results indicated that MA was associated with shorter time to first flatus (SMD $=-1.34,95 \%$ CI: -2.60 to $-0.09, P=0.04)$, while EA found no significant difference $(\mathrm{SMD}=-0.29,95 \% \mathrm{CI}:-0.63$ to $-0.05, P=0.09)$. Subgroup analyses were also conducted according to the control group, cancer type (e.g., gastric cancer and colorectal cancer), and different type of randomization; no difference in overall conclusion was observed, while heterogeneity was not resolved (Supplementary File 1). The subgroup analysis based on the type of acupuncture points used revealed that
ST-36 (Zusanli) plus SP-6 (Sanyinjiao) treatment significantly reduced the time to first flatus (SMD $=-0.62,95 \% \mathrm{CI}$ : -1.14 to $-0.10, P=0.02)$, while ST-36 treatment found no significant difference (SMD $=-1.16,95 \% \mathrm{CI}:-3.08$ to -0.75 , $P=0.23$ ) (Supplementary File 1E).

Time to First Defecation. Seven trials contributed to the combined calculation of this outcome [23-25, 27, 28, 30, 31]. Pooled results indicated that acupuncture treatment was associated with shorter time to first defecation (SMD = $-0.98,95 \%$ CI: -1.73 to $-0.22, P=0.01 ; I^{2}=94 \%$ ) (Figure 4 ). A subgroup analysis was conducted according to different types of acupuncture treatment. Our results indicated that MA was associated with shorter time to first defecation $(\mathrm{SMD}=-1.70,95 \% \mathrm{CI}:-3.33$ to $-0.06, P=0.04)$, while EA found no significant difference (SMD $=-0.34,95 \%$ CI: -0.69 to $0.00, P=0.05$ ). Subgroup analysis was also conducted according to the control group, cancer type, and type of randomization, while heterogeneity was not resolved (Supplementary File 1). The subgroup analysis based on the type of acupuncture points used revealed that ST-36 plus SP6 treatment significantly reduced the time to first defecation $(\mathrm{SMD}=-0.57,95 \% \mathrm{CI}:-0.93$ to $-0.22, P=0.03)$, while ST36 treatment found no significant difference $(\mathrm{SMD}=-1.77$, 95\% CI: -4.47 to $-0.94, P=0.20$ ) (Supplementary File $1 F$ ).

Time to First Bowel Sounds. Three trials contributed to the combined calculation of this outcome [27, 30, 31]. Pooled results indicated that acupuncture treatment was associated with a shorter time to first bowel sounds, but the difference was not statistically significant $(\mathrm{SMD}=-2.35,95 \% \mathrm{CI}:-4.74$ to $0.03, P=0.05 ; I^{2}=97 \%$ ) (Table 2 , Figure 5 ). The potential source of heterogeneity could not be explained by subgroup analysis.

Opioids Consumption and Pain Score. Four trials reported on these outcomes of opioids consumption [23, 28-30] and pain score $[23,25,28,29]$. Pooled results indicated that acupuncture treatment was associated with fewer opioids consumption $(\mathrm{SMD}=-0.38,95 \% \mathrm{CI}:-0.59$ to $-0.17, P=$ $0.0005 ; I^{2}=0 \%$ ) (Table 2, Figure 6 ). The subgroup analysis showed that EA was associated with fewer opioids consumption $(\mathrm{SMD}=-0.50,95 \% \mathrm{CI}:-0.79$ to $-0.21, P=$ $0.0008)$, while MA found no significant difference (SMD = $-0.24,95 \%$ CI: -0.56 to $0.07, P=0.13)$. The subgroup analysis based on the type of acupuncture points used revealed that ST-36 plus SP-6 treatment significantly reduced the opioids consumption (SMD $=-0.41,95 \% \mathrm{CI}:-0.64$ to $-0.18, P=0.0005)$, while ST-36 treatment found no significant difference (SMD $=-0.16,95 \% \mathrm{CI}:-0.79$ to -0.47 , $P=0.62$ ) (Supplementary File $1 G$ ). Compared with control group, acupuncture treatment was not superior on pain score $\left(\mathrm{SMD}=-0.05,95 \% \mathrm{CI}:-0.35\right.$ to $\left.0.25, P=0.74 ; I^{2}=56 \%\right)$ (Table 2, Figure 7).

Postoperative Ileus and Length of Hospital Stay. Three trials reported on outcomes of POI $[23,25,28]$ and length of hospital stay $[23,28,30]$. Compared with control group, acupuncture treatment was not superior in reducing the risk 


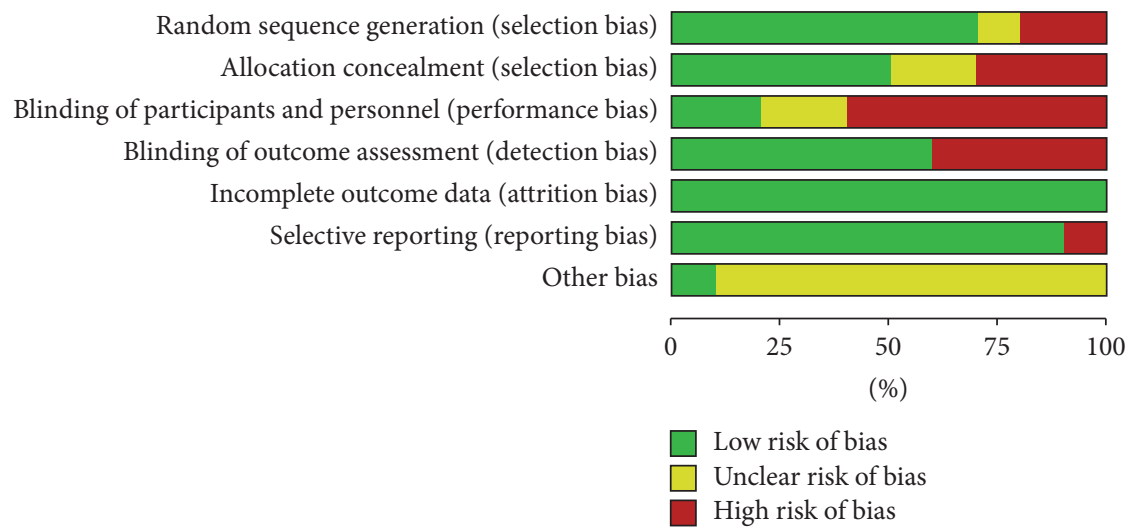

(a)

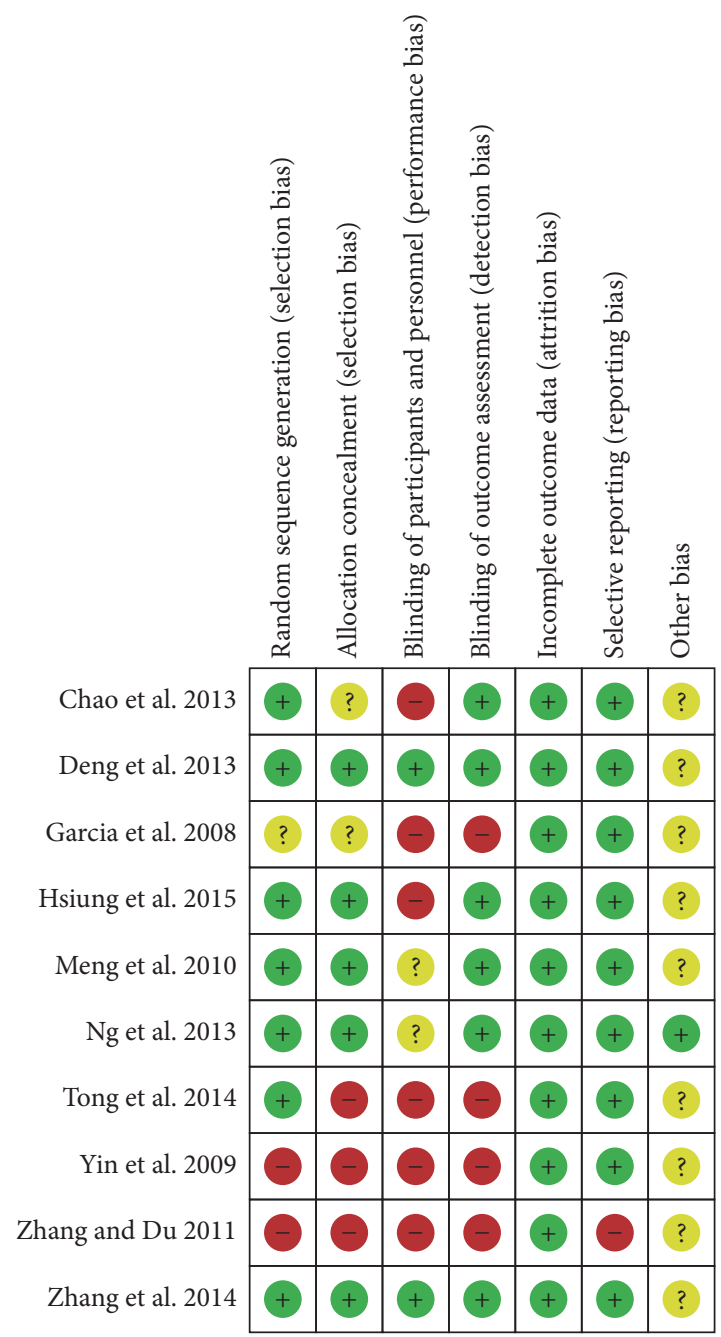

(b)

FIGURE 2: (a) Risk of bias graph: review author's judgements about each risk of bias item presented as percentages across all included studies. (b) Risk of bias summary: review author's judgements about each risk of bias item for each included study. 


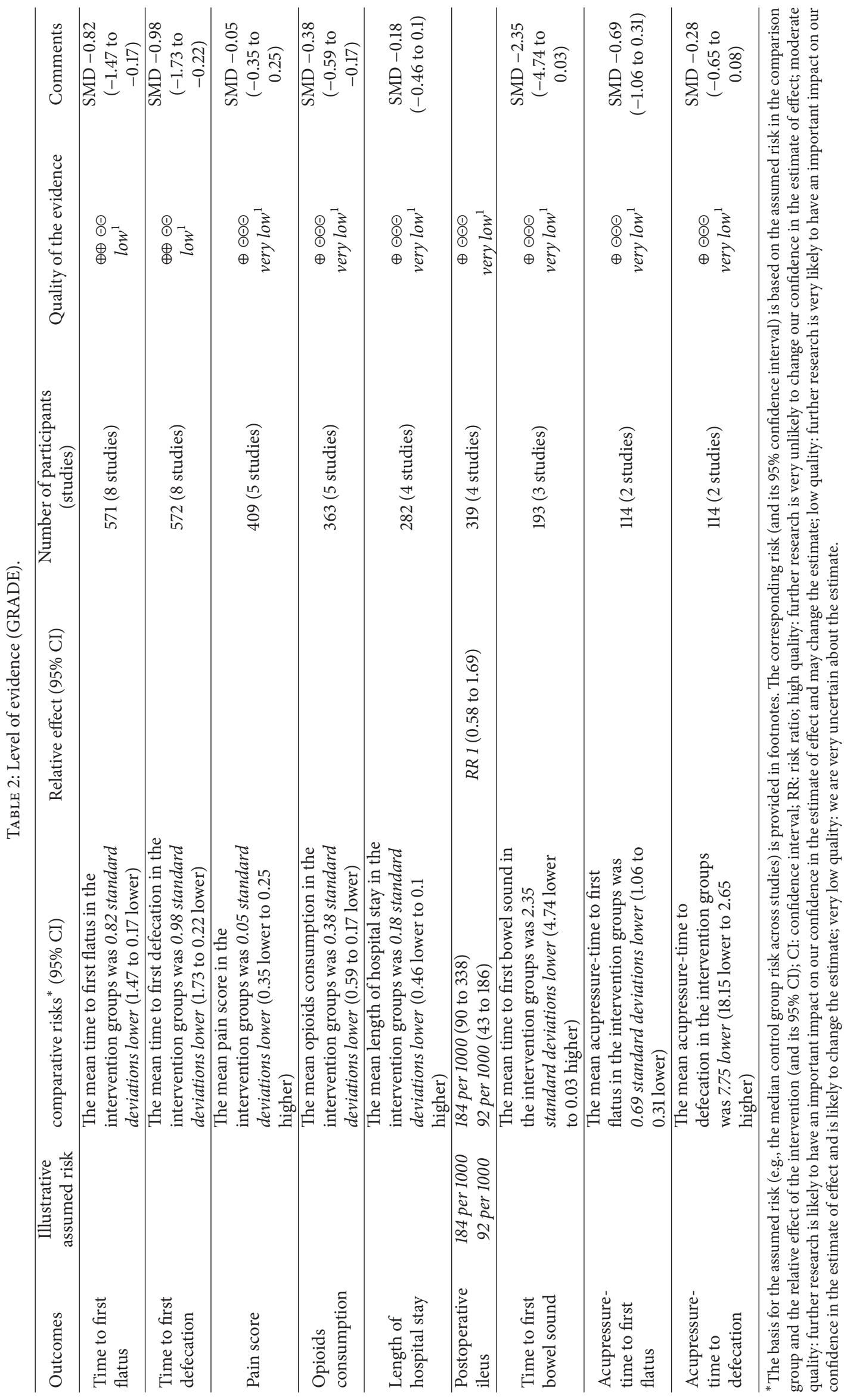




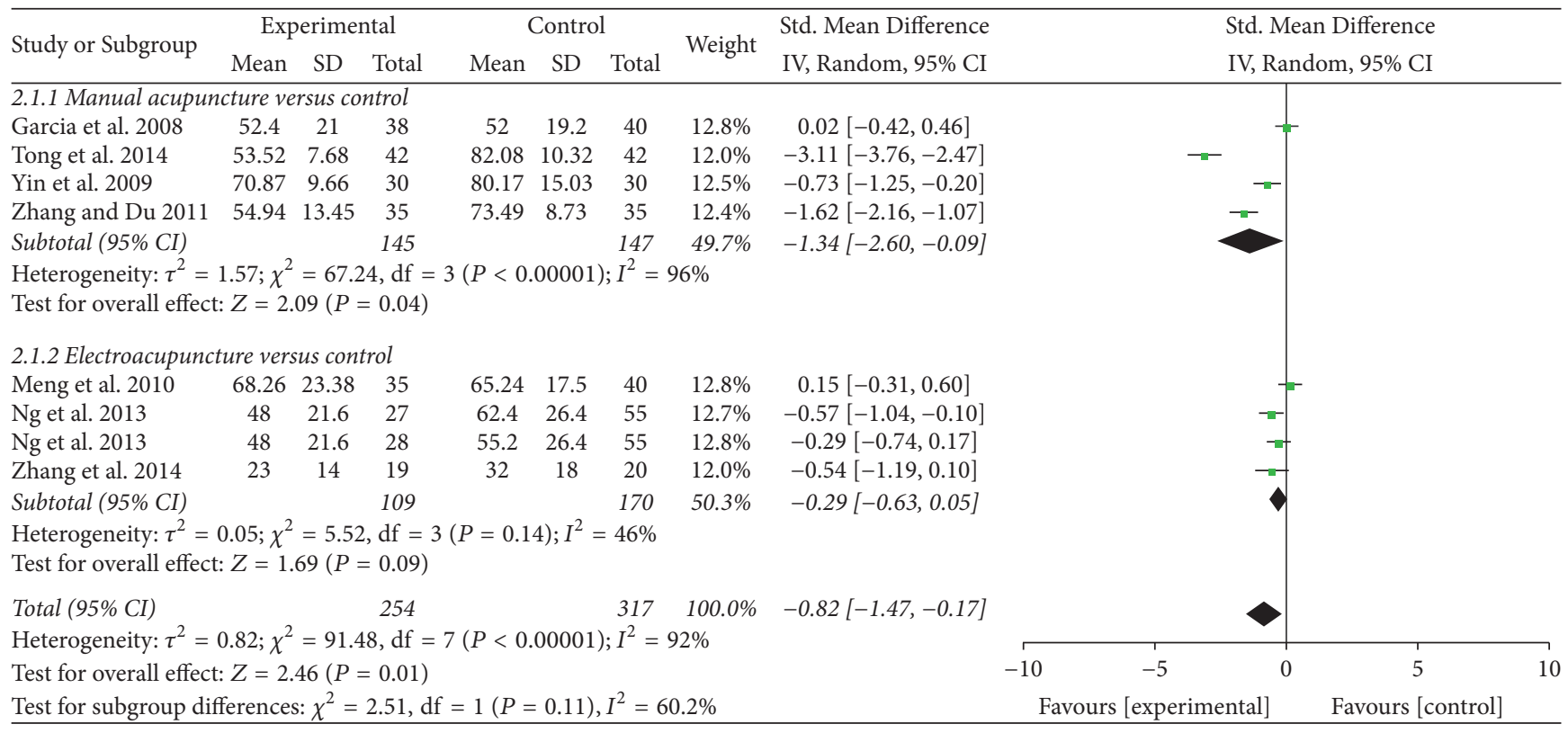

FIGURE 3: Forest plot of acupuncture treatment versus control group: time to first flatus.

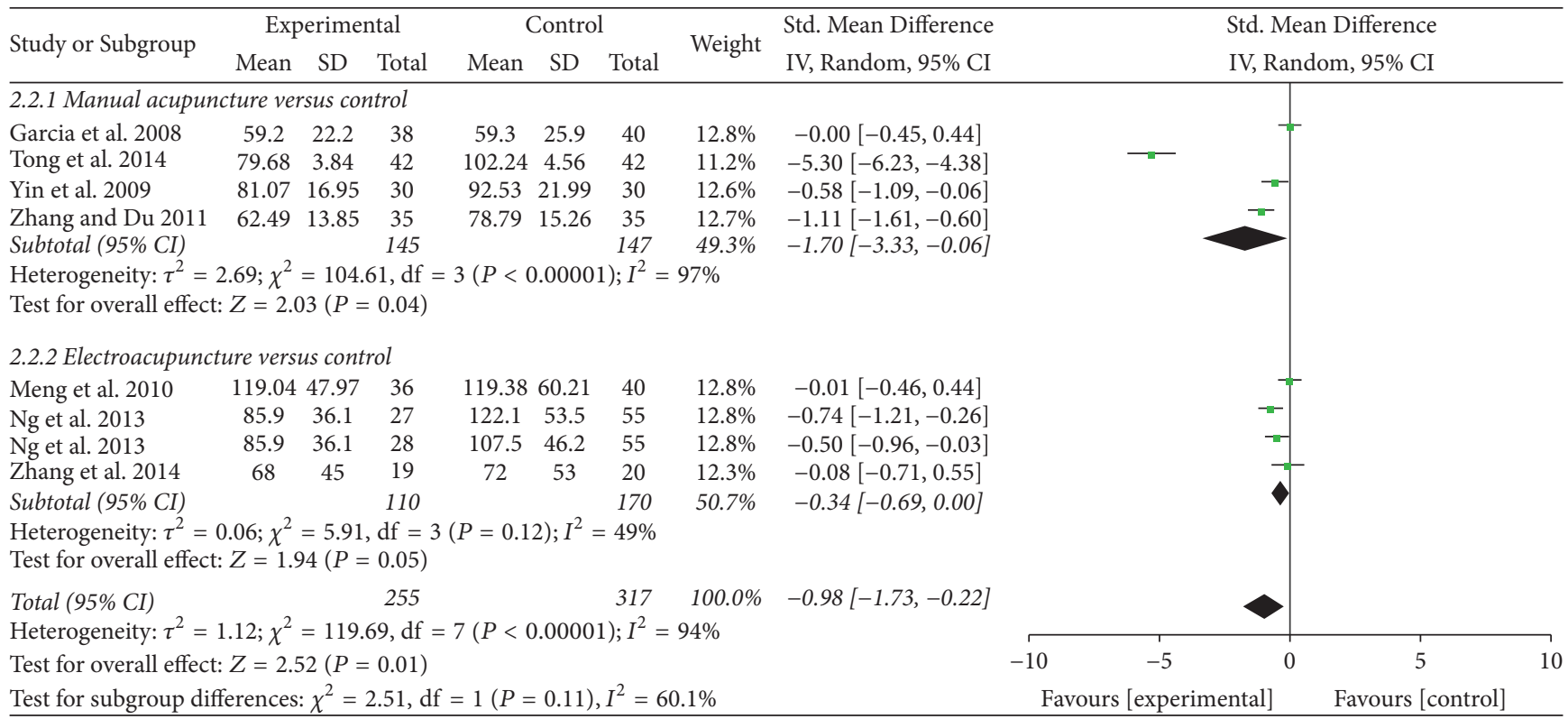

FIGURE 4: Forest plot of acupuncture treatment versus control group: time to first defecation.

of POI (RR $=0.99,95 \% \mathrm{CI}: 0.58$ to $\left.1.69, P=0.97 ; I^{2}=13 \%\right)$ (Table 2, Figure 8) and was not superior in reducing the length of hospital stay (SMD $=-0.18,95 \% \mathrm{CI}:-0.46$ to $0.10, P=$ $0.20 ; I^{2}=24 \%$ ) (Table 2, Figure 9). The subgroup analysis showed that EA was associated with shorter length of hospital stay $(\mathrm{SMD}=-0.32,95 \% \mathrm{CI}:-0.61$ to $0.03, P=0.03)$.

\subsubsection{Effects of Acupressure Treatment}

Time to First Flatus and Time to First Defecation. Two trials contributed to the combined calculation of these outcomes $[26,32]$. Pooled results indicated that acupressure was associated with a significant benefit in time to first flatus $\left(\mathrm{SMD}=-0.69,95 \% \mathrm{CI}:-1.06\right.$ to $-0.31, P=0.0004 ; I^{2}=$ $0 \%$ ) (Table 2, Figure 10). Acupressure was associated with no significant benefits in time to first defecation $(\mathrm{SMD}=-0.28$, 95\% CI: -0.65 to $0.08, P=0.13$ ) (Table 2, Figure 11).

3.5. Adverse Events. Of the ten included RCTs, only four trials assessed adverse effects and the others did not. One trial [23] mentioned that no serious adverse events related to acupuncture therapy occurred. Three trials $[25,28,32]$ reported that 


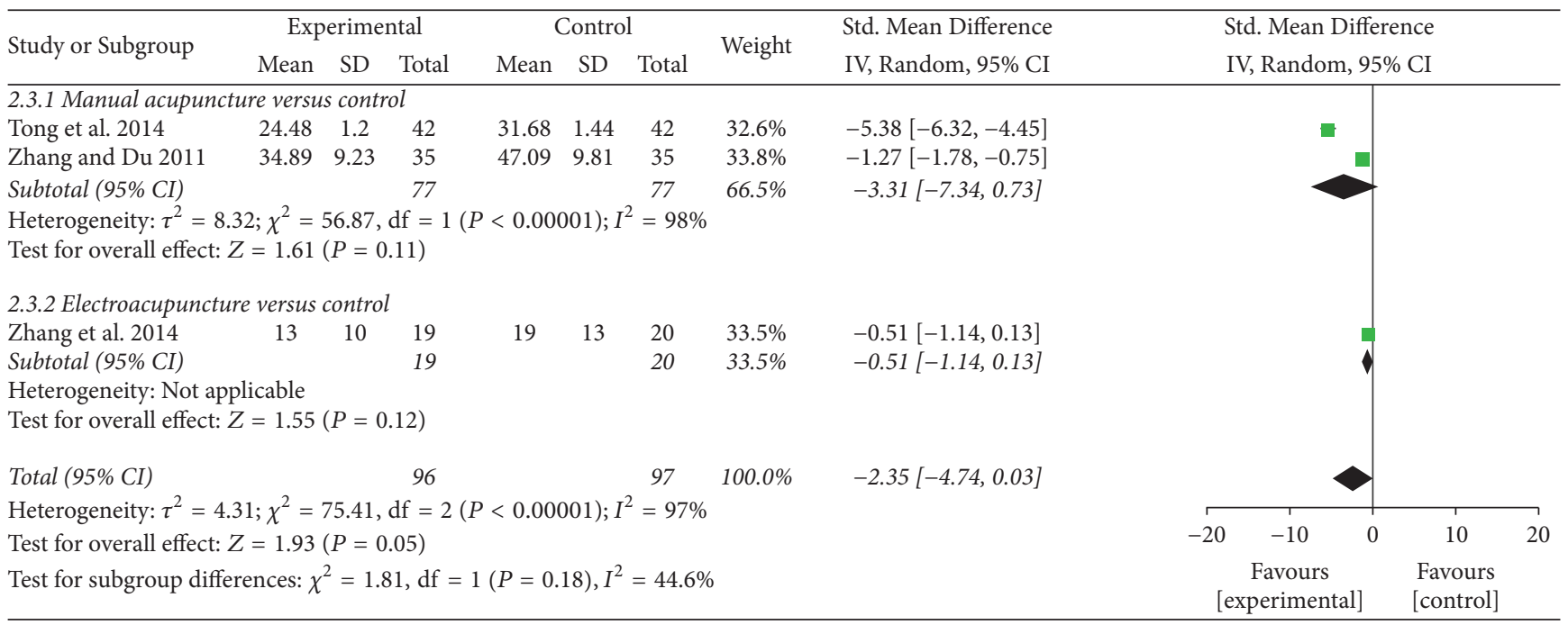

FIGURE 5: Forest plot of acupuncture treatment versus control group: time to first bowel sound.

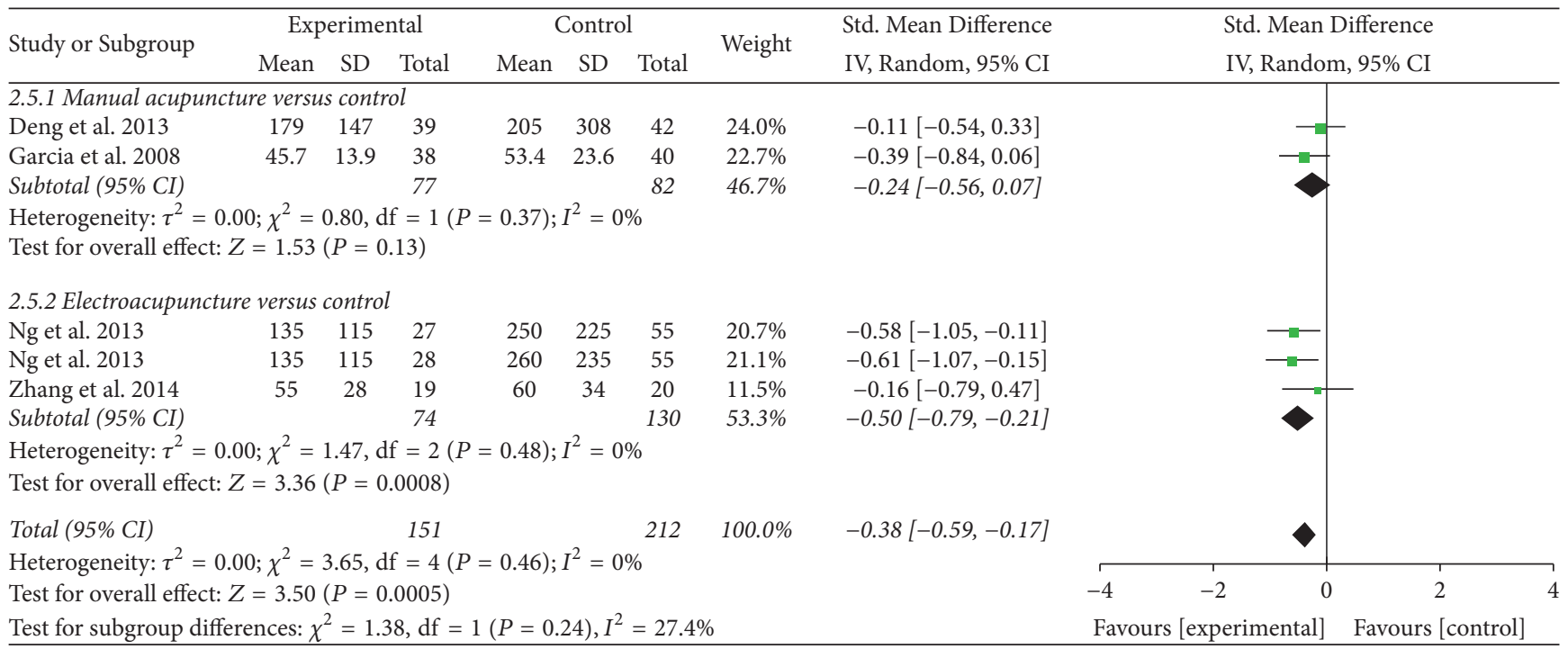

FIGURE 6: Forest plot of acupuncture treatment versus control group: opioids consumption.

there were no adverse events related to acupuncture therapy reported.

3.6. Sensitivity Analysis. A sensitivity analysis was undertaken by removing one study at a time to find the explanation and investigating the influence of each study on the overall risk estimate. The result of time to first flatus and time to first defecation was not significantly affected by omission of a single study (Supplementary File 2). The combined SMD per change in time to first flatus ranged from -0.49 (95\% CI: -0.92 to -0.07 ) to -0.96 (95\% CI: -1.66 to -0.21 ); time to first defecation ranged from -0.42 (95\% CI: -0.74 to -0.11 ) to -1.12 (95\% CI: -1.99 to -0.25$)$. After carefully reviewing included trials, we can reasonably conclude that these heterogeneities cannot impair the overall effect size of acupuncture treatment.

3.7. Publication Bias. Funnel plots were not applied to investigate the publication bias since the number of included studies was limited (less than 10 trials).

3.8. Cumulative Meta-Analysis. Cumulative meta-analysis indicated that a statistically significant effect of acupuncture treatment on the time to first flatus was first observed after the publication of the 7 th trial in 2014, while the time to first defecation was first observed after the 5th trial in 2013. The subsequent trials did not increase the precision of the 


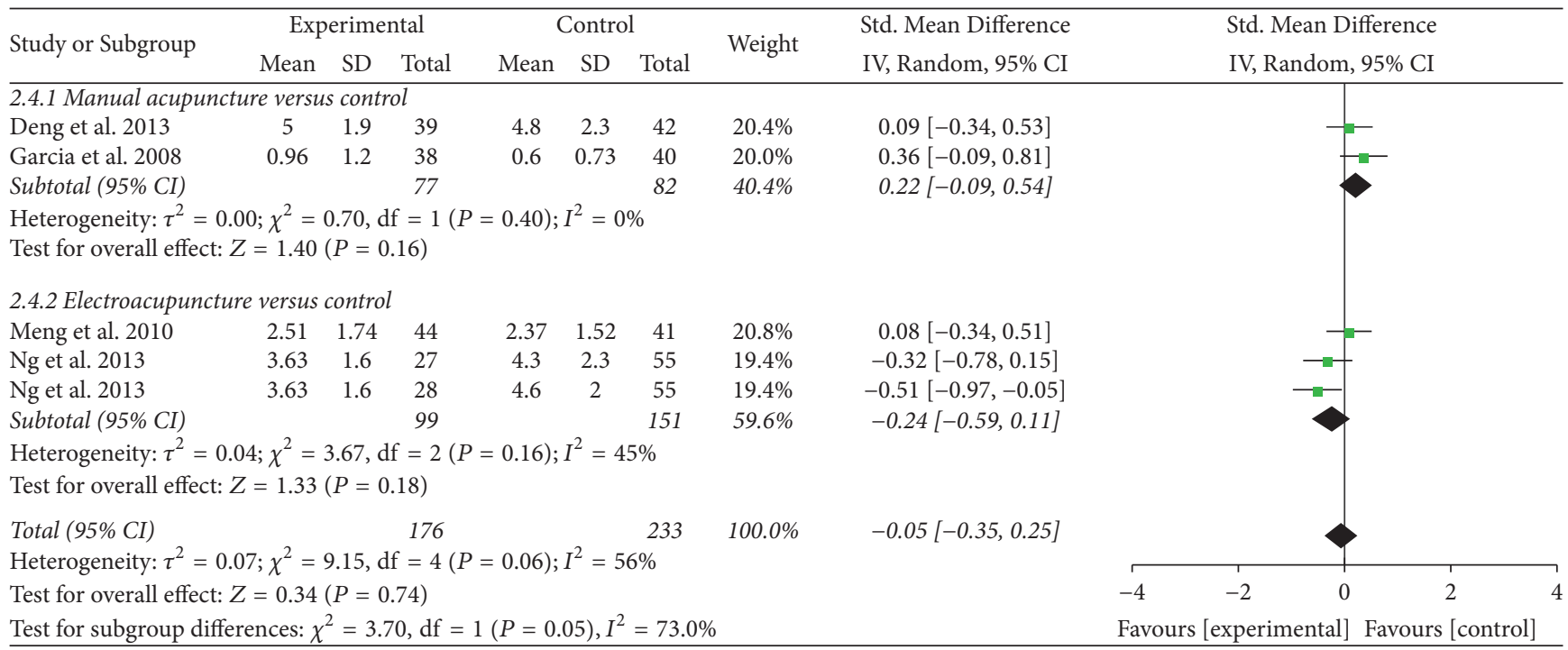

FIGURE 7: Forest plot of acupuncture treatment versus control group: pain score.

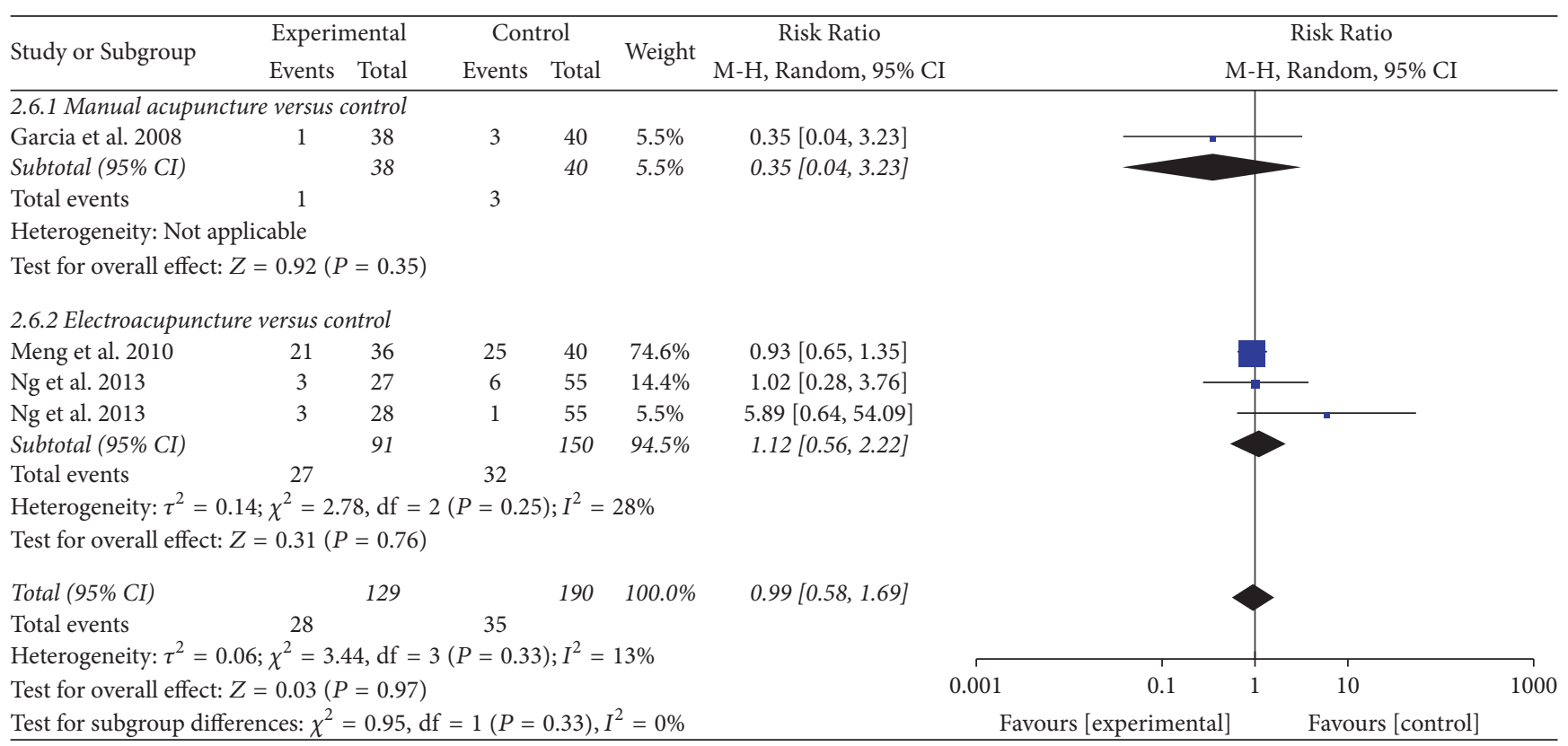

FIGURE 8: Forest plot of acupuncture treatment versus control group: risk of POI.

pooled results, and no change occurred in the trend of the intervention effect (Supplementary File 2).

\section{Discussion}

4.1. Summary of Evidence. The current meta-analysis of 10 trials demonstrated that acupuncture was associated with reduced time to first flatus, time to first defecation, and fewer opioids consumption in cancer patients after surgery. Moreover, acupressure was more effective than other comparators for the time to first flatus. These findings indicated that acupuncture and acupressure tend to have better benefits for the recovery of bowel function than sham acupuncture/acupressure, no acupuncture/acupressure, or other active control therapy (such as acupuncture plus Chinese herbal medicine versus Chinese herbal medicine). However, the results might differ by the type of acupuncture and acupuncture point. Subgroup analysis suggested that MA was more effective in the improvement of time to first flatus and time to first defecation, while EA was associated with reduced the length of hospital stay. ST-36 plus SP-6 treatment was associated with reduced time to first flatus, time to first defecation, and opioids consumption, while ST-36 treatment found no significant difference. However, these subgroups contain other acupuncture points except ST-36 and SP-6. Due to the confounding factors of other acupuncture points, 


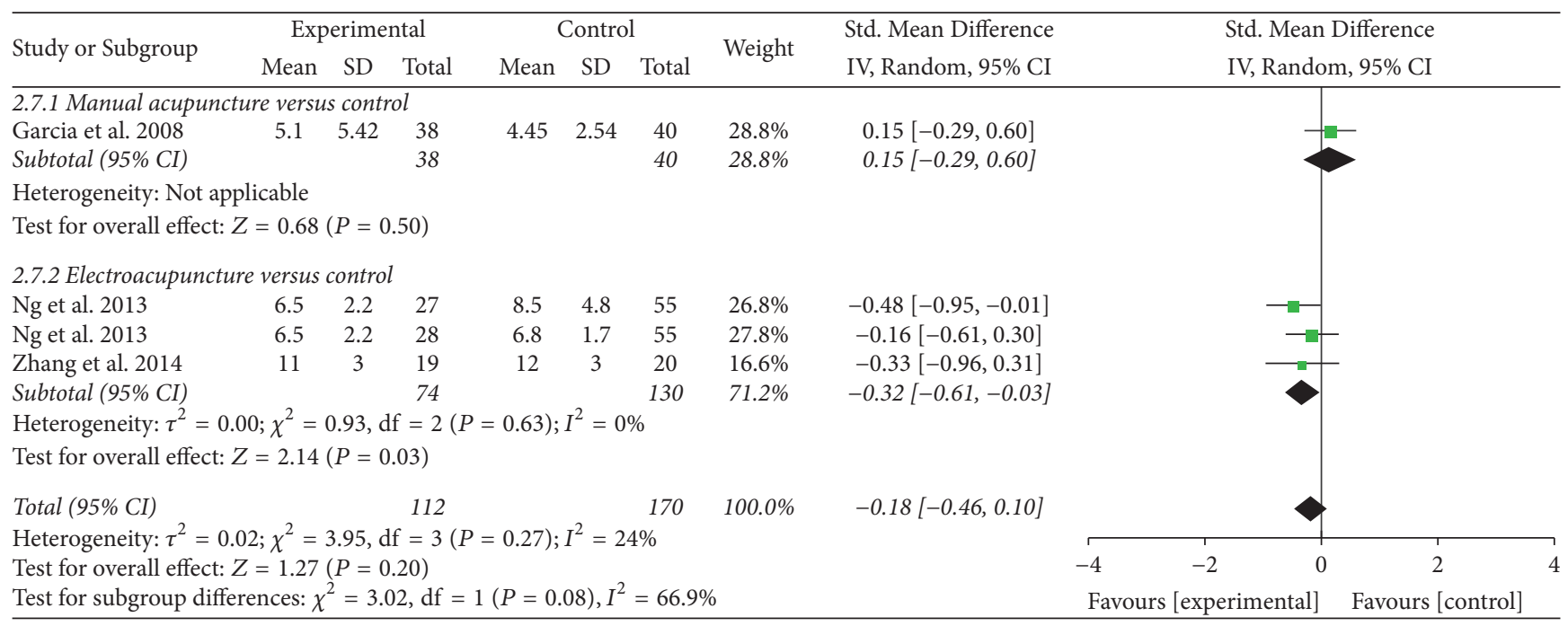

FIGURE 9: Forest plot of acupuncture treatment versus control group: length of hospital stay.

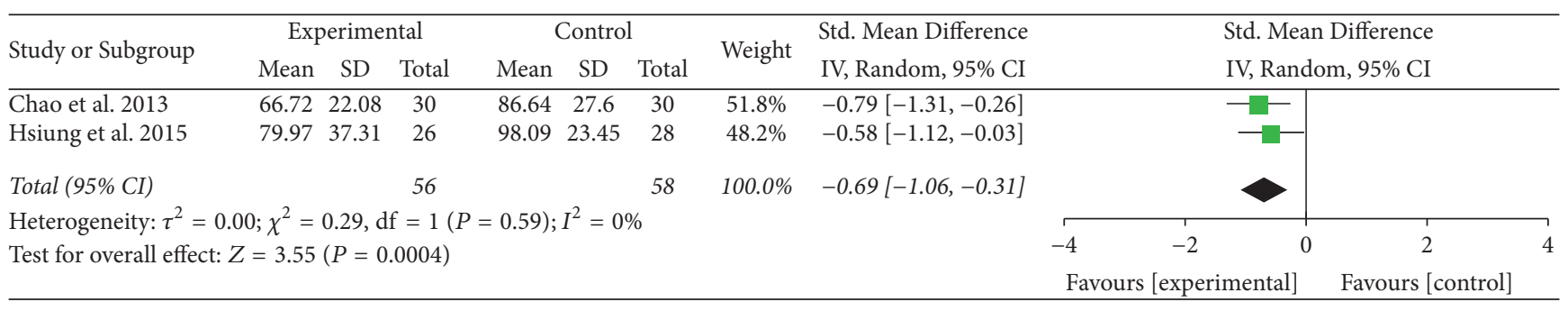

FIGURE 10: Forest plot of acupressure treatment versus control group: time to first flatus.

\begin{tabular}{|c|c|c|c|c|c|c|c|c|c|c|c|c|c|c|}
\hline \multirow{2}{*}{ Study or Subgroup } & \multicolumn{3}{|c|}{ Experimental } & \multicolumn{3}{|c|}{ Control } & \multirow{2}{*}{ Weight } & \multirow{2}{*}{$\begin{array}{c}\text { Std. Mean Difference } \\
\text { IV, Fixed, 95\% CI }\end{array}$} & \multirow{2}{*}{\multicolumn{6}{|c|}{$\begin{array}{c}\text { Std. Mean Difference } \\
\text { IV, Fixed, 95\% CI }\end{array}$}} \\
\hline & Mean & SD & Total & Mean & SD & Total & & & & & & & & \\
\hline Chao et al. 2013 & 114 & 28.08 & 30 & 126 & 47.04 & 30 & $52.6 \%$ & $-0.31[-0.81,0.20]$ & & & & & & \\
\hline Hsiung et al. 2015 & 108.55 & 23.29 & 26 & 114.63 & 22.65 & 28 & $47.4 \%$ & $-0.26[-0.80,0.28]$ & & & & & & \\
\hline Total $(95 \%$ CI) & & & 56 & & & 58 & $100.0 \%$ & $-0.28[-0.65,0.08]$ & & & & & & \\
\hline \multicolumn{9}{|c|}{ Heterogeneity: $\chi^{2}=0.01, \mathrm{df}=1(P=0.91) ; I^{2}=0 \%$} & -4 & -2 & 0 & & 2 & 4 \\
\hline \multicolumn{9}{|c|}{ Test for overall effect: $Z=1.51(P=0.13)$} & & $\exp$ & & Favours & [control] & \\
\hline
\end{tabular}

FIGURE 11: Forest plot of acupressure treatment versus control group: time to first defecation.

the comprehensive conclusion of whether the result varied by acupuncture points is difficult to draw. In the sensitive analysis of primary outcomes, omitting any trial cannot impair the overall effect size of acupuncture.

Time to first flatus and time to first defecation are essential components in the assessment of gastrointestinal dysfunction and POI [7]. These outcomes are commonly accepted as the clinical endpoint of POI and valuable indexes for evaluating the effect of interventions [39]. The decreased time to first flatus and time to first defecation after interventions indicate the improvement of bowel function. However, currently available evidence for these indicators is very limited. Nine RCTs with a small sample size assessed these measurements, while no trials mention the long-term effect of acupuncture and acupressure. Moreover, substantial heterogeneity was a limitation of the analysis of acupuncture for these measurements. The variety of intervention and control groups may have contributed to heterogeneity. But subgroup analysis (according to the type of intervention, type of control group, and type of cancer) did not totally explain the heterogeneity. It should also be emphasized through that time to first flatus is difficult to assess accurately (self-reported outcomes) [40]. Without clear measures, separating the specific effects of needling from nonspecific therapeutic factors is challenging.

The optimal management of postoperative pain is an important component of postoperative recovery after surgical resection [41]. Anesthetics and opioid analgesics used in the postoperative period are believed to be major factors that 
contribute to delayed bowel function [41]. The reduction of VAS pain score and opioids consumption can also indicate the effect of interventions for bowel function. The results of this meta-analysis indicated that acupuncture could significantly decrease the use of opioids consumption, which is consistent with previous studies supporting the relationship between acupuncture and postoperative pain [42]. The heterogeneity was acceptable in the analysis of these measurements. However, the included four trials did not report data of dropouts and intention-to-treat analysis.

In addition, POI due to oncological surgery greatly influences patients' length of hospital stay [8]. The overall effect of risk of POI and length of hospital stay showed no significant difference compared with control groups (sham acupuncture and no acupuncture). Subgroup analysis supported a benefit of EA on the length of hospital stay. Two trials of EA exactly reported the process of the randomization and allocation concealment. Meanwhile, the heterogeneity was low in the analysis of these measurements. The finding was remarkable, but the evidence was still limited.

Since the quality of outcomes determined by GRADE approach was low to very low, the currently available evidence is inadequate to make a firm conclusion on the issue of effectiveness by using acupuncture and acupressure.

4.2. Potential Mechanism of Acupuncture. POI is a common gastrointestinal problem following surgery. Risk factors for POI include long time of surgery, hemorrhage, and extensive manipulations of abdominal cavity [43]. Since these conditions frequently occur in cancer patients, it can be expected that cancer patients would have more risk of developing POI than others. A variety of strategies have been proposed to reduce POI. Nonpharmacological techniques such as laparoscopic surgery and fast-track recovery programs are primarily aimed at smaller incisions, reduced pain, and improved perioperative care management; pharmacological agents such as cyclooxygenase 2 (COX-2) inhibitors, ghrelin agonists, and opioid agonists focus on decreasing inflammation or acting on $\mu$-opioid-receptor [14]. Since no strategies have been completely successful in preventing postoperative ileus, complementary and alternative therapies may be used as an adjunct to symptom management $[14,44,45]$.

Acupuncture is a widely used complementary and alternative treatment for alleviating symptoms and improving the quality of life in cancer patients, and it is considered an effective adjuvant approach for various gastrointestinal diseases $[19,20]$. A commonly used clinical end point of POI was the time to recovery of gastrointestinal function. Although the exact mechanism of how acupuncture may reduce POI in cancer patients is unknown, acupuncture has been shown to improve gastrointestinal dysrhythmia [46, 47], secretion [48], accelerate solid gastric emptying [49], and restore impaired gastrointestinal motility mediated via the cholinergic pathway [50]. Extensive research indicates that acupuncture has the potential to treat gastrointestinal disorders by regulating the gastrointestinal barrier, visceral sensitivity, and the brain-gut axis [51]. A pilot study found that acupuncture has the potential effect on reducing the duration of POI after gastric cancer surgery, by the earlier recovery of small bowel movement [52]. A recent preclinical study has revealed that EA administered at ST36 promotes the recovery time of POI by exciting nucleus of the solitary tract neurons [18]. Thus, for an intervention that is effective and of low cost and has few side effects, acupuncture is worth clinical generalization and application.

4.3. Comparison with Other Studies. A previous systematic review evaluated the use of acupuncture broadly for cancer care but did not provide a determined result for POI in cancer patients. In this study, potential available RCTs were assessed as having a high risk of bias, and no trials met their inclusion criteria [19]. Another systematic review suggested that acupuncture for cancer patients with POI presented no significant difference within the intergroups [21]. Indeed, no meta-analysis has been performed before. The current updated meta-analysis reveals some new findings. First, due to the similar mechanism of action, we included additional intervention type of acupressure to assess its effect on POI in cancer patients. The results of this meta-analysis suggested that acupuncture was associated with shorter time to first flatus and time to defecation compared with all control interventions, which differed from the previous reviews. Second, this review managed to identify more RCTs to explore any available evidence. Two previous RCTs demonstrated no significant difference of acupuncture compared with sham or no acupuncture in cancer patients with POI. Meng et al. [25] acknowledged that the use of epidural anesthesia might have diminished the possible effects of acupuncture mediated by neural mechanisms; Garcia et al. [23] did not mention the randomization and allocation concealment. Then, $\mathrm{Ng}$ et al. [28] conducted a larger and more rigorous randomized study, excluded patients who had received anesthesia or analgesia, and minimized the risk of randomization and allocation concealment. They found that EA reduced the duration of POI, and opioids consumption, compared with sham or no acupuncture in colorectal cancer. Most other results of recent RCTs are consistent with this trial. Cumulative meta-analysis indicated that the subsequent trials did not increase the precision of the pooled positive results.

4.4. Limitations. This meta-analysis has a number of limitations. One obvious limitation is the diversity of control interventions, acupuncture manipulation (needle location, treatment duration, and frequency); therefore, definite conclusions regarding these confounders could not be drawn. High-quality trials with large sample size are needed to provide stronger evidence. Further, a lack of standardized study design and outcome measures might affect the reliability of findings. Future trials should follow the CONSORT statement or STRICTA recommendations [34]. In addition, the databases considered in this review were limited to English and Chinese literature which might contribute to bias and limit our findings.

\section{Conclusion}

In conclusion, the present findings suggest that acupuncture and acupressure demonstrate better efficacy for cancer 
patients on the recovery of bowel function compared with control interventions. However, the effect size of acupuncture treatment might be limited by the inferior quality of included trials. Further rigorous studies with adequate power are needed to determine the effectiveness of acupuncture in cancer patients with bowel dysfunction and POI.

\section{Conflicts of Interest}

The authors declare that they have no conflicts of interest.

\section{Acknowledgments}

This work was partially supported by the State Administration of Traditional Chinese Medicine (no. 201307006) and the International S\&T Cooperation Program Office of China (no. 2013DFA32540).

\section{Supplementary Materials}

Supplementary file 1. Subgroup analysis. Supplemental file 1A-D: primary outcomes of acupuncture treatment: subgroup analysis by cancer type and various control interventions. Supplemental file 1A: Forest plots of outcome "Time to first flatus" Subgroup: cancer type. Supplemental file 1B: Forest plots of outcome "Time to first defecation" Subgroup: cancer type. Supplemental file 1C: Forest plots of outcome "Time to first flatus" Subgroup: control type. Supplemental file 1D: Forest plots of outcome "Time to first defecation" Subgroup: control type. Supplemental file 1E-G: primary outcomes of acupuncture treatment: subgroup analysis by acupuncture points. Supplemental file 1E: Forest plots of outcome "Time to first flatus" Subgroup: acupuncture points. Supplemental file 1F: Forest plots of outcome "Time to first defecation" Subgroup: acupuncture points. Supplemental file 1G: Forest plots of outcome "Opioids consumption" Subgroup: acupuncture points. Supplementary file 2. Sensitivity analysis and Cumulative meta-analysis. Supplementary file 2. Sensitivity analysis and Cumulative meta-analysis. Supplemental file 2A-B. Sensitivity analyses of the comparison between acupuncture and control group. a: Time to first flatus; b: Time to first defecation. Supplemental file 2A. Sensitivity analysis for time to first flatus between acupuncture and control group. Supplemental file 2B. Sensitivity analysis for time to first defecation between acupuncture and control group. Supplemental file 2C-D. Cumulative metaanalysis of the comparison between acupuncture and control group. c Time to first flatus; d Time to first defecation. Supplemental file 2C: Cumulative meta-analysis of the comparison between acupuncture and control group: time to first flatus. Supplemental file 2D: Cumulative meta-analysis of the comparison between acupuncture and control group: time to first defecation. Supplementary file 3. Reporting quality of RCTs based on STRICTA. (Supplementary Materials)

\section{References}

[1] H. B. Neuman, D. Schrag, C. Cabral et al., "Can differences in bowel function after surgery for rectal cancer be identified by the European organization for research and treatment of cancer quality of life instrument?" Annals of Surgical Oncology, vol. 14, no. 5, pp. 1727-1734, 2007.

[2] B. S. Langenhoff, P. F. M. Krabbe, T. Wobbes, and T. J. M. Ruers, "Quality of life as an outcome measure in surgical oncology," British Journal of Surgery, vol. 88, no. 5, pp. 643-652, 2001.

[3] A. J. Bauer and G. E. Boeckxstaens, "Mechanisms of postoperative ileus," Neurogastroenterology \& Motility, vol. 16, no. 2, pp. 54-60, 2004.

[4] B. G. Wolff, F. Michelassi, T. M. Gerkin et al., "Alvimopan, a novel, peripherally acting mu opioid antagonist: Results of a multicenter, randomized, double-blind, placebo-controlled, phase iii trial of major abdominal surgery and postoperative ileus," Annals of Surgery, vol. 240, no. 4, pp. 728-735, 2004.

[5] Z. Wu, G. S. A. Boersema, A. Dereci, A. G. Menon, J. Jeekel, and J. F. Lange, "Clinical endpoint, early detection, and differential diagnosis of postoperative ileus: a systematic review of the literature," European Surgical Research, vol. 54, no. 3-4, pp. 127138, 2015.

[6] B. Person and S. D. Wexner, "The management of postoperative ileus," Current Problems in Surgery, vol. 43, no. 1, pp. 6-65, 2006.

[7] R. Vather, S. Trivedi, and I. Bissett, "Defining postoperative ileus: results of a systematic review and global survey," Journal of Gastrointestinal Surgery, vol. 17, no. 5, pp. 962-972, 2013.

[8] H. Kehlet, "Postoperative ileus - An update on preventive techniques," Nature Clinical Practice Gastroenterology \& Hepatology, vol. 5, no. 10, pp. 552-558, 2008.

[9] R. Merkow, M. Ju, and J. Chung, "Underlying reasons associated with hospital readmission following surgery in the united states," Journal of Vascular Surgery, vol. 313, no. 5, pp. 483-495, 2015.

[10] A. Venara, M. Neunlist, K. Slim et al., "Postoperative ileus: pathophysiology, incidence, and prevention," Journal of Visceral Surgery, vol. 153, no. 6, pp. 439-446, 2016.

[11] R. Vather, G. O'Grady, I. P. Bissett, and P. G. Dinning, "Postoperative ileus: Mechanisms and future directions for research," Clinical and Experimental Pharmacology and Physiology, vol. 41, no. 5, pp. 358-370, 2014.

[12] H. Kehlet, "Fast-track colorectal surgery," The Lancet, vol. 371, no. 9615, pp. 791-793, 2008.

[13] T. Asgeirsson, K. I. El-Badawi, A. Mahmood, J. Barletta, M. Luchtefeld, and A. J. Senagore, "Postoperative ileus: it costs more than you expect," Journal of the American College of Surgeons, vol. 210, no. 2, pp. 228-231, 2010.

[14] S. H. W. Van Bree, A. Nemethova, C. Cailotto, P. J. GomezPinilla, G. Matteoli, and G. E. Boeckxstaens, "New therapeutic strategies for postoperative ileus," Nature Reviews Gastroenterology \& Hepatology, vol. 9, no. 11, pp. 675-683, 2012.

[15] X.-M. You, X.-S. Mo, L. Ma et al., "Randomized Clinical Trial Comparing Efficacy of Simo Decoction and Acupuncture or Chewing Gum Alone on Postoperative Ileus in Patients with Hepatocellular Carcinoma after Hepatectomy," Medicine (United States), vol. 94, no. 45, p. e1968, 2015.

[16] U. O. Gustafsson, M. J. Scott, W. Schwenk et al., "Guidelines for perioperative care in elective colonic surgery: enhanced Recovery After Surgery (ERAS) Society recommendations," Clinical Nutrition, vol. 31, no. 6, pp. 783-800, 2012.

[17] K. Mortensen, M. Nilsson, K. Slim et al., "Consensus guidelines for enhanced recovery after gastrectomy: Enhanced Recovery after Surgery (ERAS ${ }^{\circledR}$ ) Society recommendations," British Journal of Surgery, vol. 101, no. 10, pp. 1209-1229, 2014. 
[18] J.-F. Fang, J.-Q. Fang, X.-M. Shao et al., "Electroacupuncture treatment partly promotes the recovery time of postoperative ileus by activating the vagus nerve but not regulating local inflammation," Scientific Reports, vol. 7, Article ID 39801, 2017.

[19] M. Kay Garcia, J. Mcquade, R. Haddad et al., "Systematic review of acupuncture in cancer care: a synthesis of the evidence," Journal of Clinical Oncology, vol. 31, no. 7, pp. 952-960, 2013.

[20] Institute NC, Acupuncture ( $p d q(r))$ : Health professional version, Pdq cancer information summaries, 2002.

[21] W.-L. Lian, M.-Q. Pan, D.-H. Zhou, and Z.-J. Zhang, "Effectiveness of acupuncture for palliative care in cancer patients: a systematic review," Chinese Journal of Integrative Medicine, vol. 20, no. 2, pp. 136-147, 2014.

[22] D. Moher, A. Liberati, J. Tetzlaff, and D. G. Altman, "Preferred reporting items for systematic reviews and meta-analyses: the PRISMA statement," PLoS Medicine, vol. 6, no. 7, Article ID e1000097, 2009.

[23] M. K. Garcia, J. M. Skibber, M. A. Rodriguez-Bigas et al., "Acupuncture to prevent prolonged postoperative ileus: A randomized controlled trial," Medical Acupuncture, vol. 20, no. 2, pp. 83-88, 2008.

[24] S.-H. Yin, Y.-Q. Du, and B. Liu, "Clinical study on acupuncture combined with medication in restoration of gastrointestinal functions for postoperative patients with gastric cancer," Chinese acupuncture and moxibustion, vol. 29, no. 6, pp. 459-462, 2009.

[25] Z. Q. Meng, M. K. Garcia, J. S. Chiang et al., "Electroacupuncture to prevent prolonged postoperative ileus: a randomized clinical trial," World Journal of Gastroenterology, vol. 16, no. 1, pp. 104-111, 2010.

[26] H.-L. Chao, S.-J. Miao, P.-F. Liu et al., “The beneficial effect of ST-36 (Zusanli) acupressure on postoperative gastrointestinal function in patients with colorectal cancer," Oncology Nursing Forum, vol. 40, no. 2, pp. E61-E68, 2013.

[27] S. Zhang and Y. Du, "Effects of warming needle moxibustion on improvement of gastrointestinal and immune function in patients with postoperation of colorectal cancer," Chinese Acupuncture and Moxibustion, vol. 31, no. 6, pp. 513-517, 2011.

[28] S. S. M. Ng, W. W. Leung, T. W. C. Mak et al., "Electroacupuncture reduces duration of postoperative ileus after laparoscopic surgery for colorectal cancer," Gastroenterology, vol. 144, no. 2, pp. 307.e1-313.e1, 2013.

[29] G. Deng, W. D. Wong, J. Guillem et al., "A phase II, randomized, controlled trial of acupuncture for reduction of postcolectomy ileus," Annals of Surgical Oncology, vol. 20, no. 4, pp. 1164-1169, 2013.

[30] Z. Zhang, C. Wang, Q. Li et al., "Electroacupuncture at ST36 accelerates the recovery of gastrointestinal motility after colorectal surgery: a randomised controlled trial," Acupuncture in Medicine, vol. 32, no. 3, pp. 223-226, 2014.

[31] W. Y. Tong et al., "Effects of acupuncture on gastrointestinal function in postoperative rectal cancer patients," Guiding Journal of Traditional Chinese Medicine and Pharmacy, vol. 20, no. 12, pp. 39-41, 2014.

[32] W.-T. Hsiung, Y.-C. Chang, M.-L. Yeh, and Y.-H. Chang, "Acupressure improves the postoperative comfort of gastric cancer patients: a randomised controlled trial," Complementary Therapies in Medicine, vol. 23, no. 3, pp. 339-346, 2015.

[33] S. H. W. Van Bree, W. A. Bemelman, M. W. Hollmann et al., "Identification of clinical outcome measures for recovery of gastrointestinal motility in postoperative ileus," Annals of Surgery, vol. 259, no. 4, pp. 708-714, 2014.
[34] H. MacPherson, D. G. Altman, R. Hammerschlag et al., "Revised STandards for Reporting Interventions in Clinical Trials of Acupuncture (STRICTA): extending the CONSORT statement," PLoS Medicine, vol. 7, no. 6, Article ID e1000261, 2010.

[35] J. P. T. Higgins, D. G. Altman, P. C. Gøtzsche et al., "The Cochrane Collaboration's tool for assessing risk of bias in randomised trials," British Medical Journal, vol. 343, no. 7829, Article ID d5928, 2011.

[36] G. H. Guyatt, A. D. Oxman, G. E. Vist et al., "GRADE: an emerging consensus on rating quality of evidence and strength of recommendations," British Medical Journal, vol. 336, no. 7650, pp. 924-926, 2008.

[37] J. P. T. Higgins, S. G. Thompson, J. J. Deeks, and D. G. Altman, "Measuring inconsistency in meta-analyses," British Medical Journal, vol. 327, no. 7414, pp. 557-560, 2003.

[38] M. Egger, G. D. Smith, M. Schneider, and C. Minder, "Bias in meta-analysis detected by a simple, graphical test," British Medical Journal, vol. 315, pp. 629-634, 1997.

[39] P. Mattei and J. L. Rombeau, "Review of the pathophysiology and management of postoperative ileus," World Journal of Surgery, vol. 30, no. 8, pp. 1382-1391, 2006.

[40] M. Maffezzini, F. Campodonico, G. Canepa, G. Gerbi, and D. Parodi, "Current perioperative management of radical cystectomy with intestinal urinary reconstruction for muscle-invasive bladder cancer and reduction of the incidence of postoperative ileus," Surgical Oncology, vol. 17, no. 1, pp. 41-48, 2008.

[41] M. Tan, L. S.-C. Law, and T. J. Gan, "Optimizing pain management to facilitate Enhanced Recovery After Surgery pathways," Canadian Journal of Anesthesia, vol. 62, no. 2, pp. 203-218, 2016.

[42] C. Hu, H. Zhang, and W. Wu, "Acupuncture for pain management in cancer: a systematic review and meta-analysis," Evidence-Based Complementary and Alternative Medicine, vol. 2016, Article ID 1720239, 13 pages, 2016.

[43] A. Artinyan, J. W. Nunoo-Mensah, S. Balasubramaniam et al., "Prolonged postoperative ileus - Definition, risk factors, and predictors after surgery," World Journal of Surgery, vol. 32, no. 7, pp. 1495-1500, 2008.

[44] G. Deng, B. R. Cassileth, and K. S. Yeung, "Complementary therapies for cancer-related symptoms," The Journal of Supportive Oncology, vol. 2, no. 5, pp. 419-426, 2004.

[45] A. Bonacchi, A. Toccafondi, A. Mambrini et al., "Complementary needs behind complementary therapies in cancer patients," Psycho-Oncology, vol. 24, no. 9, pp. 1124-1130, 2015.

[46] N. Danielli Miller, E. Schiff, E. Ben-Arye et al., "Benefits of acupuncture for diabetic gastroparesis: a comparative preliminary study," Acupuncture in medicine: journal of the British Medical Acupuncture Society, vol. 32, no. 2, pp. 139-145, 2014.

[47] J. Yin, J. Chen, and J. D. Z. Chen, "Ameliorating effects and mechanisms of electroacupuncture on gastric dysrhythmia, delayed emptying, and impaired accommodation in diabetic rats," American Journal of Physiology-Gastrointestinal and Liver Physiology, vol. 298, no. 4, pp. G563-G570, 2010.

[48] H. Tada, M. Fujita, M. Harris et al., "Neural mechanism of acupuncture-induced gastric relaxations in rats," Digestive Diseases and Sciences, vol. 48, no. 1, pp. 59-68, 2003.

[49] C.-P. Wang, C.-H. Kao, W.-K. Chen, W.-Y. Lo, and C.-L. Hsieh, "A single-blinded, randomized pilot study evaluating effects of electroacupuncture in diabetic patients with symptoms suggestive of gastroparesis," The Journal of Alternative and Complementary Medicine, vol. 14, no. 7, pp. 833-839, 2008. 
[50] D. Luo, S. Liu, X. Xie, and X. Hou, "Electroacupuncture at acupoint ST-36 promotes contractility of distal colon via a cholinergic pathway in conscious rats," Digestive Diseases and Sciences, vol. 53, no. 3, pp. 689-693, 2008.

[51] H. Li, T. He, Q. Xu et al., "Acupuncture and regulation of gastrointestinal function," World Journal of Gastroenterology, vol. 21, no. 27, pp. 8304-8313, 2015.

[52] H.-D. Chae, M.-A. Kwak, and I.-H. Kim, "Effect of acupuncture on reducing duration of postoperative ileus after gastrectomy in patients with gastric cancer: A pilot study using sitz marker," The Journal of Alternative and Complementary Medicine, vol. 22, no. 6, pp. 465-472, 2016. 


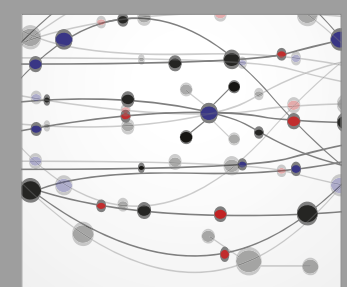

The Scientific World Journal
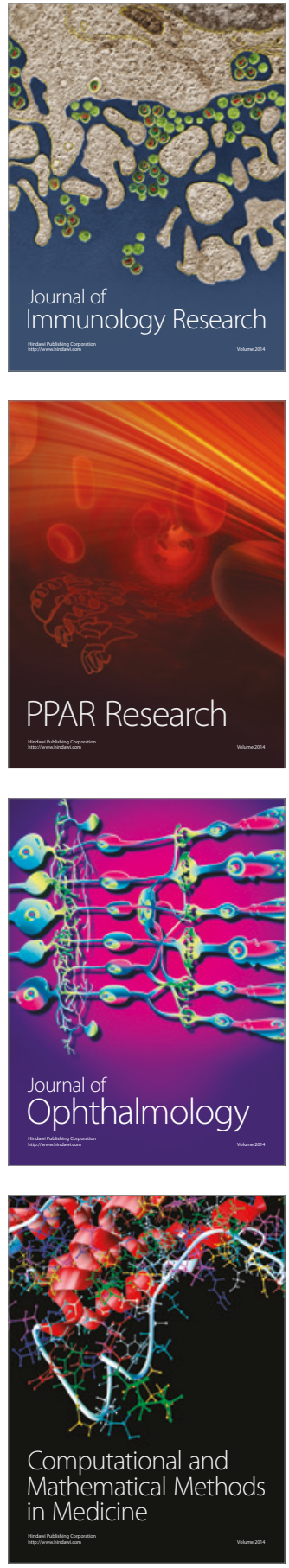

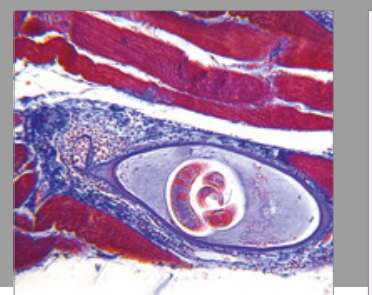

Gastroenterology Research and Practice
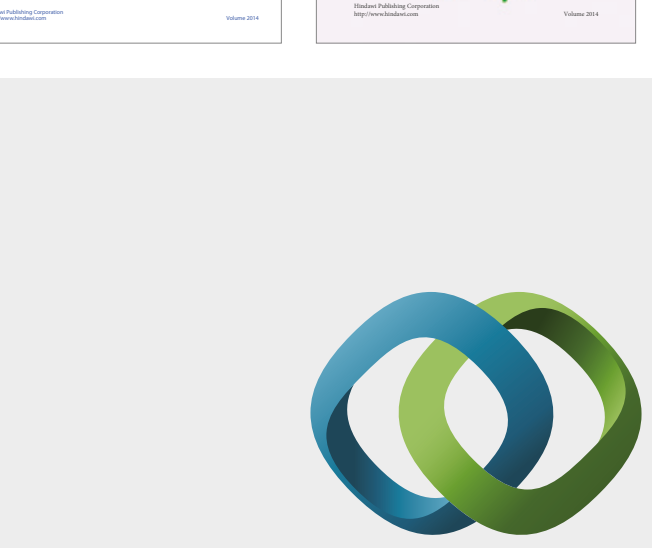

\section{Hindawi}

Submit your manuscripts at

https://www.hindawi.com
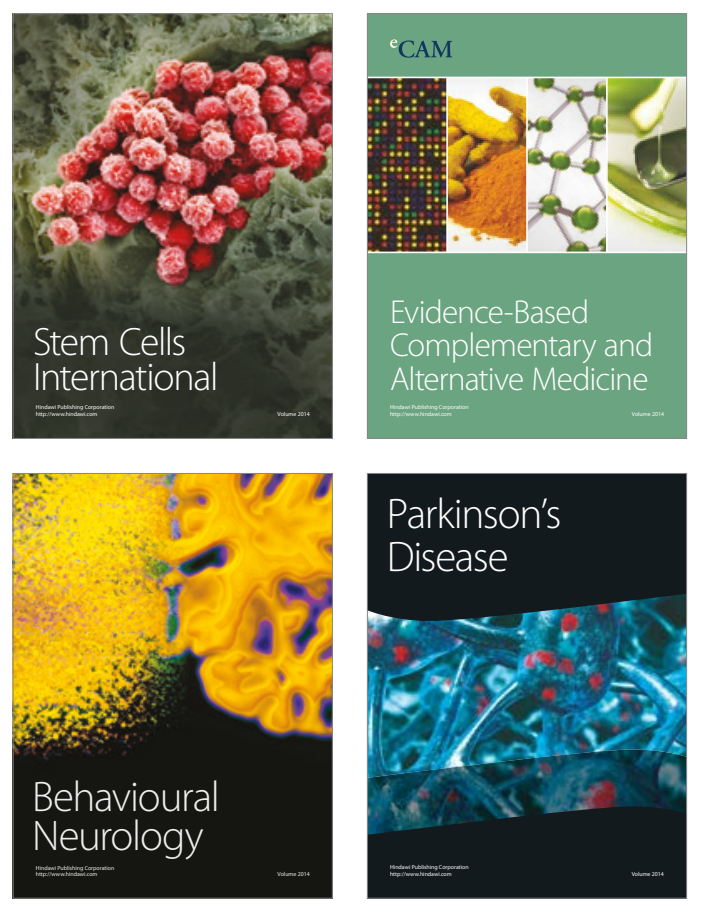
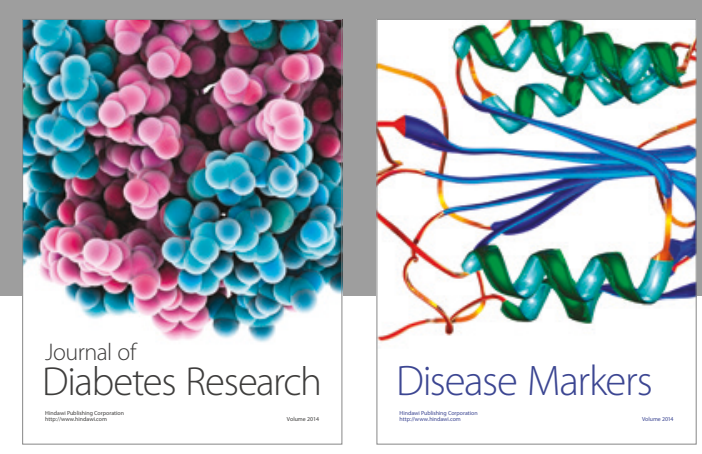

Disease Markers
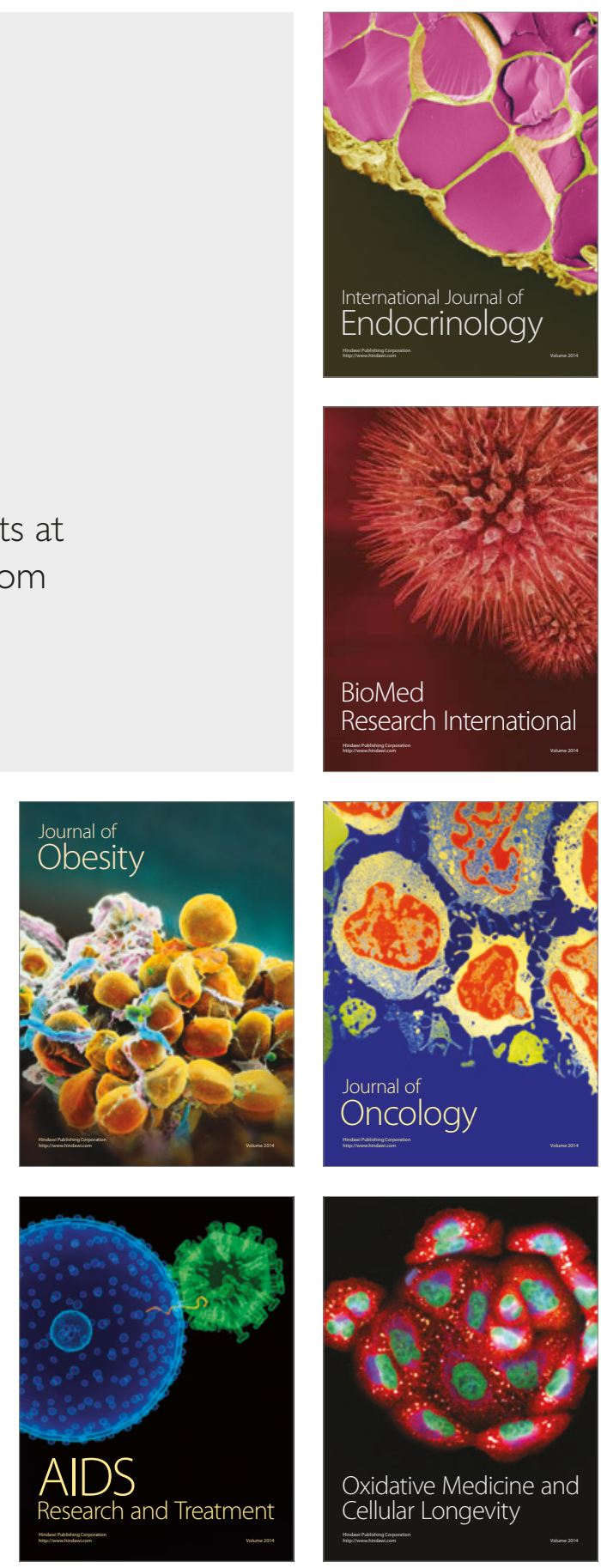